\title{
Time to Onset of Response to Pitolisant for the Treatment of Excessive Daytime Sleepiness and Cataplexy in Patients With Narcolepsy: An Analysis of Randomized, Placebo-Controlled Trials
}

\author{
Nathaniel F. Watson ${ }^{1}$. Craig W. Davis² Donna Zarycranski $^{2} \cdot$ Ben Vaughn $^{3} \cdot$ Jeffrey M. Dayno ${ }^{2}$ Yves Dauvilliers $^{4,5,6}$. \\ Jean-Charles Schwartz ${ }^{7}$
}

Accepted: 26 September 2021 / Published online: 25 November 2021

(c) The Author(s) 2021

\begin{abstract}
Background Pitolisant is approved in the USA and Europe for the treatment of excessive daytime sleepiness or cataplexy in adults with narcolepsy.

Objective Analyses evaluated the time to onset of clinical response during treatment with pitolisant.

Methods Data were obtained from two randomized, double-blind, 7-week or 8-week, placebo-controlled studies (HARMONY 1, HARMONY CTP). Study medication was individually titrated to a maximum dose of pitolisant 35.6 $\mathrm{mg} /$ day and then remained stable. Efficacy assessments included the Epworth Sleepiness Scale and weekly rate of cataplexy (calculated from patient diaries). Onset of clinical response was defined as the first timepoint at which there was statistical separation between pitolisant and placebo.

Results The analysis included 61 patients in HARMONY 1 (pitolisant, $n=31$; placebo, $n=30$ ) and 105 patients in HARMONY CTP (pitolisant, $n=54$; placebo, $n=51$ ). Onset of clinical response began at week 2 (HARMONY 1) or week 3 (HARMONY CTP) for the mean change in Epworth Sleepiness Scale score, and week 2 (HARMONY CTP) or week 5 (HARMONY 1) for the mean change in weekly rate of cataplexy, with further improvements observed in pitolisant-treated patients through the end of treatment. The percentage of treatment responders was significantly greater with pitolisant vs placebo beginning at week 3 for excessive daytime sleepiness (defined as an Epworth Sleepiness Scale score reduction $\geq 3$ ) and week 2 for cataplexy (defined as a $\geq 50 \%$ reduction in weekly rate of cataplexy [HARMONY CTP]).

Conclusions Onset of clinical response for excessive daytime sleepiness and/or cataplexy was generally observed within the first 2-3 weeks of pitolisant treatment in patients with narcolepsy.

ClinicalTrials.gov identifier NCT01067222 (February 2010), NCT01800045 (February 2013).
\end{abstract}

Nathaniel F. Watson

nwatson@uw.edu

1 Department of Neurology, University of Washington School of Medicine, Seattle, WA, USA

2 Harmony Biosciences, LLC, Plymouth Meeting, PA, USA

3 Rho, Durham, NC, USA

4 National Reference Center for Narcolepsy, Montpellier, France

5 Gui-de-Chauliac Hospital, CHU Montpellier, University of Montpellier, Montpellier, France

6 Institute for Neurosciences of Montpellier INM, INSERM, University of Montpellier, Montpellier, France

7 Bioprojet Pharma, Paris, France

\section{Key Points}

Pitolisant treats excessive daytime sleepiness and cataplexy in patients with narcolepsy, and the onset of clinical response is often seen within 2-3 weeks of initiating treatment.

Inter-individual variation is observed in the time to the onset of clinical response, which may be related to pitolisant dose during titration.

Consider titrating the pitolisant dose to $35.6 \mathrm{mg} /$ day, within the limits of tolerability. 


\section{Introduction}

Narcolepsy is a disorder of sleep-wake state instability characterized primarily by excessive daytime sleepiness (EDS) and symptoms of rapid eye movement (REM) sleep dysregulation, such as cataplexy [1,2]. Excessive daytime sleepiness is a required component of the diagnostic criteria for narcolepsy, whereas cataplexy - the sudden brief loss of muscle tone usually triggered by emotion-is a hallmark symptom of narcolepsy type $1[3,4]$. In patients with narcolepsy, EDS and cataplexy can be debilitating, with detrimental effects on school performance, employment (opportunities, productivity, and income), social functioning, and emotional well-being [5-10].

Deficiency in hypocretin (also known as orexin) signaling, resulting from the selective loss of hypocretin neurons from the dorsolateral hypothalamus, has been identified as the cause of narcolepsy with cataplexy (narcolepsy type 1); the etiology of narcolepsy without cataplexy (narcolepsy type 2) remains unclear [11]. Immune-mediated processes appear to underlie the destruction of hypocretin-producing neurons in narcolepsy type 1 and may also be involved in some cases of narcolepsy type 2 [12, 13]. In the absence of viable hypocretin-replacement therapies or hypocretinreceptor agonists, treatments for narcolepsy are symptomdriven and aim to promote wakefulness and suppress cataplexy and other symptoms of REM-sleep disturbance (e.g., hypnagogic hallucinations, sleep paralysis) $[14,15]$.

In the past several years, there has been increased recognition of the important functions of histamine in the regulation of sleep and wakefulness $[16,17]$. Histaminergic neurons originate in the hypothalamus, the primary brain region for regulating sleep-wake timing and stability, and project widely throughout the brain $[16,18,19]$. Histamine activates cortical and subcortical neurons to promote and sustain wakefulness and also suppresses non-REM and REM sleep via inhibition of neurons in sleep-promoting brain regions [18-22]. Findings from preclinical research show that histamine is important for normal sleep-wake behavior and may stabilize sleep-wake transitions [16, 23].

Pitolisant is a selective histamine $3\left(\mathrm{H}_{3}\right)$-receptor antagonist/inverse agonist that increases the synthesis and release of histamine in the brain via competitive binding to presynaptic $\mathrm{H}_{3}$ autoreceptors [21, 24]. Pitolisant also binds to $\mathrm{H}_{3}$ receptors on nonhistaminergic neurons [25-27], which increases the activity of other neurotransmitters that promote wakefulness (e.g., acetylcholine, dopamine, norepinephrine) [18] and play a role in the control of cataplexy (e.g., norepinephrine, serotonin) $[28,29]$. In contrast to stimulant medications, pitolisant does not increase dopamine release in brain regions of the reward system (e.g., nucleus accumbens) in animal studies [30] and demonstrates minimal to no potential for abuse in humans [31]. The efficacy and safety of pitolisant in the treatment of patients with narcolepsy have been demonstrated in short-term, randomized, placebocontrolled trials [32, 33] and a long-term, open-label study [34]. The most common adverse reactions associated with pitolisant in placebo-controlled trials were headache $(18 \%$ of patients), insomnia (6\%), nausea (6\%), anxiety $(5 \%)$, musculoskeletal pain (5\%), and upper respiratory tract infection (5\%) [35]. Pitolisant is approved by the US Food and Drug Administration for the treatment of EDS or cataplexy in adult patients with narcolepsy [35] and by the European Medicines Agency [36] for the treatment of narcolepsy with or without cataplexy in adults.

The recommended clinical dosing regimen for pitolisant includes a 3-week titration period, with a maximum daily dosage of $35.6 \mathrm{mg}[35,36] .{ }^{1}$ There may be variability among patients in the time needed to achieve a clinical response to a medication, especially for compounds that require dose titration. Thus, information about the time to the onset of therapeutic effect can improve management of patients' expectations regarding narcolepsy treatments, aid in medication selection, and optimize dosing. The objective of this analysis was to evaluate the time to the onset of clinical response with pitolisant for the treatment of EDS or cataplexy in adults with narcolepsy.

\section{Methods}

Data for this analysis were obtained from two randomized, double-blind, 7-week or 8-week, placebo-controlled studies of adults with narcolepsy in which pitolisant was administered up to the highest recommended dose of $35.6 \mathrm{mg} /$ day (HARMONY 1, HARMONY CTP). Primary results for these studies have been published previously $[32,33]$. Both studies were conducted in accordance with the Good Clinical Practice guidelines of the International Council for Harmonisation and the ethical principles of the Declaration of Helsinki. Each study protocol was approved by an institutional review board or independent ethics committee, and all patients provided written informed consent before study enrollment.

\subsection{Patients}

These studies included adults aged 18 years or older with a diagnosis of narcolepsy with or without cataplexy

\footnotetext{
${ }^{1}$ Pitolisant doses reported in some previous publications have included the hydrochloride salt $(5 \mathrm{mg}, 10 \mathrm{mg}, 20 \mathrm{mg}, 40 \mathrm{mg})$. The doses presented here reflect the pitolisant base, consistent with US Food and Drug Administration guidance $(4.45 \mathrm{mg}, 8.9 \mathrm{mg}, 17.8 \mathrm{mg}$, $35.6 \mathrm{mg}$ ). In Europe, doses reflect the pitolisant base but are rounded to one fewer decimal place $(4.5 \mathrm{mg}, 9 \mathrm{mg}, 18 \mathrm{mg}, 36 \mathrm{mg}$ ).
} 
(HARMONY 1) or narcolepsy with cataplexy (HARMONY CTP) according to International Classification of Sleep Disorders, 2nd edition criteria. All patients were experiencing EDS, with an Epworth Sleepiness Scale (ESS) score of at least 14 (HARMONY 1) or 12 (HARMONY CTP) at baseline. Patients in HARMONY CTP also were required to have at least three cataplexy attacks per week at baseline. Patients were excluded from study participation if they had another condition that may cause EDS (e.g., sleep-disordered breathing with apnea index $\geq 10 / \mathrm{h}$ or apnea/hypopnea index $\geq 15 / \mathrm{h}$, periodic limb movement disorder with arousal index $\geq 10 /$ h, shift work sleep disorder, circadian rhythm disorder), current or recent (within 1 year) substance abuse or dependence, significant cardiovascular abnormality, severe hepatic or renal impairment, psychiatric or neurological disorders, or other active clinically significant illness.

\subsection{Study Procedures}

Stimulants and other wake-promoting medications (e.g., methylphenidate, amphetamines, modafinil) were discontinued prior to study baseline. Other anti-cataplectic medications (e.g., sodium oxybate, selective serotonin reuptake inhibitors, serotonin-norepinephrine reuptake inhibitors) were permitted, provided the dose had been stable for 1 month prior to screening and was not altered during the study. Tricyclic antidepressants, which have antagonist activity at $\mathrm{H}_{1}$ receptors, were not permitted.

Study medication was individually titrated based on efficacy and tolerability; however, the dosing regimen differed slightly between studies (Table 1). The dose of study medication started at $8.9 \mathrm{mg} /$ day in HARMONY 1 and $4.45 \mathrm{mg} /$ day in HARMONY CTP and was flexibly titrated over a 3-week period; the dose selected by the investigator at the beginning of week 4 (up to $35.6 \mathrm{mg} / \mathrm{day}$ ) remained stable for the subsequent 4 (HARMONY CTP) or 5 (HARMONY 1) weeks of treatment. Both studies included a 1-week placebo washout period after the end of treatment.

Efficacy was evaluated using the ESS [37], frequency of cataplexy attacks, and Clinical Global Impression of Change
(CGI-C) [38] for EDS and for cataplexy. The ESS was administered at baseline (prior to the first dose of study medication) and at all post-baseline study visits (weeks, 2, 3, 7, and 8 for HARMONY 1; weeks 2, 3, 6, and 7 for HARMONY CTP); the CGI-C was administered at all post-baseline study visits. Treatment response for EDS was based on the ESS score and defined in two ways: a score reduction of $\geq 3$ from baseline and a final score of $\leq 10$. Response on the CGI-C was defined by ratings of "very much" or "much" improved. The weekly rate of cataplexy (WRC) attacks was calculated using information recorded in patient diaries. Treatment response for cataplexy was calculated for HARMONY CTP using two thresholds: $\geq 25 \%$ and $\geq 50 \%$ reductions in WRC. This categorical variable was not analyzed for HARMONY 1 because of the small sample size of patients with cataplexy at baseline. Onset of clinical response was defined as the first assessment timepoint at which there was a statistically significant difference between pitolisant and placebo in the mean change from baseline (for ESS score and WRC) or percentage of responders (for EDS response, cataplexy response, and CGI-C). Adverse events (AEs) were assessed throughout the studies and coded using the Medical Dictionary for Regulatory Activities.

\subsection{Statistical Analysis}

These analyses included patients in the intent-to-treat population who were randomly assigned to receive pitolisant or placebo. The intent-to-treat population consisted of patients who received one or more doses of study medication and completed one or more post-baseline assessments. Changes from baseline in ESS scores and WRC were compared for pitolisant vs placebo using an analysis of covariance, with the last observation carried forward for on-treatment timepoints and observed cases for the placebo washout assessment. The percentage of responders was compared at each on-treatment timepoint for pitolisant vs placebo using Fisher's exact tests; missing values were imputed using a last observation carried forward approach. Adverse event data were summarized by week using descriptive statistics.

Table 1 Pitolisant titration and dosing

\begin{tabular}{|c|c|c|c|c|c|c|}
\hline \multirow[t]{2}{*}{ Study name } & \multicolumn{3}{|c|}{ Titration period } & \multicolumn{2}{|l|}{ Stable-dose period } & \multirow{2}{*}{$\begin{array}{l}\text { Washout } \\
1 \mathrm{wk}\end{array}$} \\
\hline & Week 1 & Week 2 & Week 3 & Dose & Duration & \\
\hline HARMONY 1 & $8.9 \mathrm{mg} / \mathrm{d}$ & $17.8 \mathrm{mg} / \mathrm{d}$ & $\begin{array}{l}8.9 \mathrm{mg} / \mathrm{d}, 17.8 \\
\mathrm{mg} / \mathrm{d} \text {, or } 35.6 \\
\mathrm{mg} / \mathrm{d}\end{array}$ & $\begin{array}{l}8.9 \mathrm{mg} / \mathrm{d}, 17.8 \mathrm{mg} / \mathrm{d} \text {, or } \\
35.6 \mathrm{mg} / \mathrm{d}\end{array}$ & $5 \mathrm{wk}$ & Placebo \\
\hline HARMONY CTP & $4.45 \mathrm{mg} / \mathrm{d}$ & $8.9 \mathrm{mg} / \mathrm{d}$ & $\begin{array}{l}4.45 \mathrm{mg} / \mathrm{d}, 8.9 \\
\mathrm{mg} / \mathrm{d} \text {, or } 17.8 \\
\mathrm{mg} / \mathrm{d}\end{array}$ & $\begin{array}{l}4.45 \mathrm{mg} / \mathrm{d}, 8.9 \mathrm{mg} / \mathrm{d}, \\
17.8 \mathrm{mg} / \mathrm{d} \text {, or } 35.6 \\
\mathrm{mg} / \mathrm{d}\end{array}$ & $4 \mathrm{wk}$ & Placebo \\
\hline
\end{tabular}

$d$ day, $w k$ weeks 
Table 2 Demographic and clinical characteristics at baseline

\begin{tabular}{|c|c|c|c|c|}
\hline \multirow[t]{2}{*}{ Characteristic } & \multicolumn{2}{|l|}{ HARMONY 1} & \multicolumn{2}{|l|}{ HARMONY CTP } \\
\hline & Pitolisant $(n=31)$ & Placebo $(n=30)$ & Pitolisant $(n=54)$ & Placebo $(n=51)$ \\
\hline Age, y, median (range) & $33.0(19-65)$ & $39.5(19-75)$ & $34.0(18-64)$ & $39.0(18-66)$ \\
\hline Female sex, $n(\%)$ & $11(35.5)$ & $17(56.7)$ & $28(51.9)$ & $24(47.1)$ \\
\hline BMI, kg/m², mean (SD) & $30.4(8.3)$ & $28.2(6.0)$ & $27.2(5.2)$ & $28.8(6.0)$ \\
\hline \multicolumn{5}{|l|}{ Baseline score, mean (SD) } \\
\hline ESS score & $17.8(2.5)$ & $18.9(2.5)$ & $17.4(3.3)$ & $17.3(3.2)$ \\
\hline WRC & $10.5(12.6)^{\mathrm{a}}$ & $8.2(5.7)^{\mathrm{a}}$ & $11.7(10.0)$ & $9.6(9.5)$ \\
\hline $\begin{array}{l}\text { Concomitant anti-cataplectic } \\
\text { medication, } n(\%)^{\mathrm{b}}\end{array}$ & $13(41.9)$ & $9(30.0)$ & $4(7.4)$ & $8(15.7)$ \\
\hline
\end{tabular}

$B M I$ body mass index, ESS Epworth Sleepiness Scale, $S D$ standard deviation, $W R C$ weekly rate of cataplexy, $y$ years

${ }^{a}$ Subset of HARMONY 1 patients with cataplexy at baseline: pitolisant, $n=17$; placebo, $n=11$

${ }^{b}$ Anti-cataplectic medications (sodium oxybate, selective serotonin reuptake inhibitors and serotonin-norepinephrine reuptake inhibitors, but not tricyclic antidepressants) were permitted, provided the dose had been stable for 1 month prior to screening and was not altered during the study

\section{Results}

\subsection{Patients}

The analysis population included 61 patients from HARMONY 1 (pitolisant, $n=31$; placebo, $n=30$ ) and 105 patients from HARMONY CTP (pitolisant, $n=54$; placebo, $n=51$ ). Baseline demographics, mean ESS score, and mean WRC were similar across the two studies (Table 2). Mean ESS scores at baseline ranged from 17.3 to 18.9 , which is indicative of severe EDS [37]. In HARMONY CTP, the mean rate of cataplexy attacks at baseline was 11.7 per week in the pitolisant group and 9.6 per week in the placebo group. In the subset of HARMONY 1 patients with cataplexy at baseline (pitolisant, $n=17$; placebo, $n=11$ ), the mean rate of cataplexy attacks at baseline was 10.5 per week in the pitolisant group and 8.2 per week in the placebo group. Study medication was individually titrated over a 3-week period that allowed for flexible dosing; the dose administered at the beginning of week 4 remained stable through the end of treatment. Pitolisant was titrated to the maximum recommended dose of $35.6 \mathrm{mg} /$ day in $61.3 \%$ of patients in HARMONY 1 and $64.8 \%$ in HARMONY CTP.

\subsection{Excessive Daytime Sleepiness}

On the ESS, the least-squares (LS) mean change from baseline was significantly greater for pitolisant compared with placebo beginning at week 2 (the first post-baseline assessment) in HARMONY 1 and week 3 in HARMONY CTP (Fig. 1). All pitolisant-treated patients received $17.8 \mathrm{mg} /$ day during the second week of treatment in HARMONY 1; during the third week of HARMONY CTP, the pitolisant dose was $17.8 \mathrm{mg} /$ day in $88.9 \%$ of patients, $8.9 \mathrm{mg} /$ day in
9.3\%, and $4.45 \mathrm{mg}$ in $1.9 \%$. The significant difference in ESS score change for the pitolisant group vs the placebo group continued through the end of treatment (with the exception of a marginally significant $[p=0.05]$ difference at week 7 in HARMONY 1) and remained statistically significant after a 1-week double-blind placebo washout period in both studies (Fig. 1). At the end of treatment, the LS mean change from baseline ESS score was -6.0 for pitolisant vs -2.7 for placebo $(p=0.026)$ in HARMONY 1 and -5.6 vs $-1.6(p<0.001)$ in HARMONY CTP. More pitolisanttreated patients were classified as EDS responders at each post-baseline assessment than were patients in the placebo group, and the ESS response rate was significantly greater for pitolisant compared with placebo at the end of treatment in each study ( $p<0.05 ;$ Fig. 2 ). For response defined as an ESS score reduction of $\geq 3$, pitolisant differed significantly from placebo beginning at week 3 in both studies and continuing at each subsequent assessment except for week 7 in HARMONY 1. For a response defined as a final ESS score $\leq 10$, separation from placebo was observed beginning at week 3 in HARMONY 1 but not until week 7 in HARMONY CTP. On the CGI-C for EDS, more patients were considered by the investigator to be "much" or "very much" improved with pitolisant vs placebo; however, the difference only reached statistical significance in HARMONY CTP, at the end of treatment (Fig. 3).

\subsection{Cataplexy}

In HARMONY CTP, the frequency of cataplexy attacks was significantly reduced for pitolisant compared with placebo beginning at week 2 and continued through the end of treatment (Fig. 4). All pitolisant-treated patients were receiving $8.9 \mathrm{mg} /$ day during the second week of treatment 
A
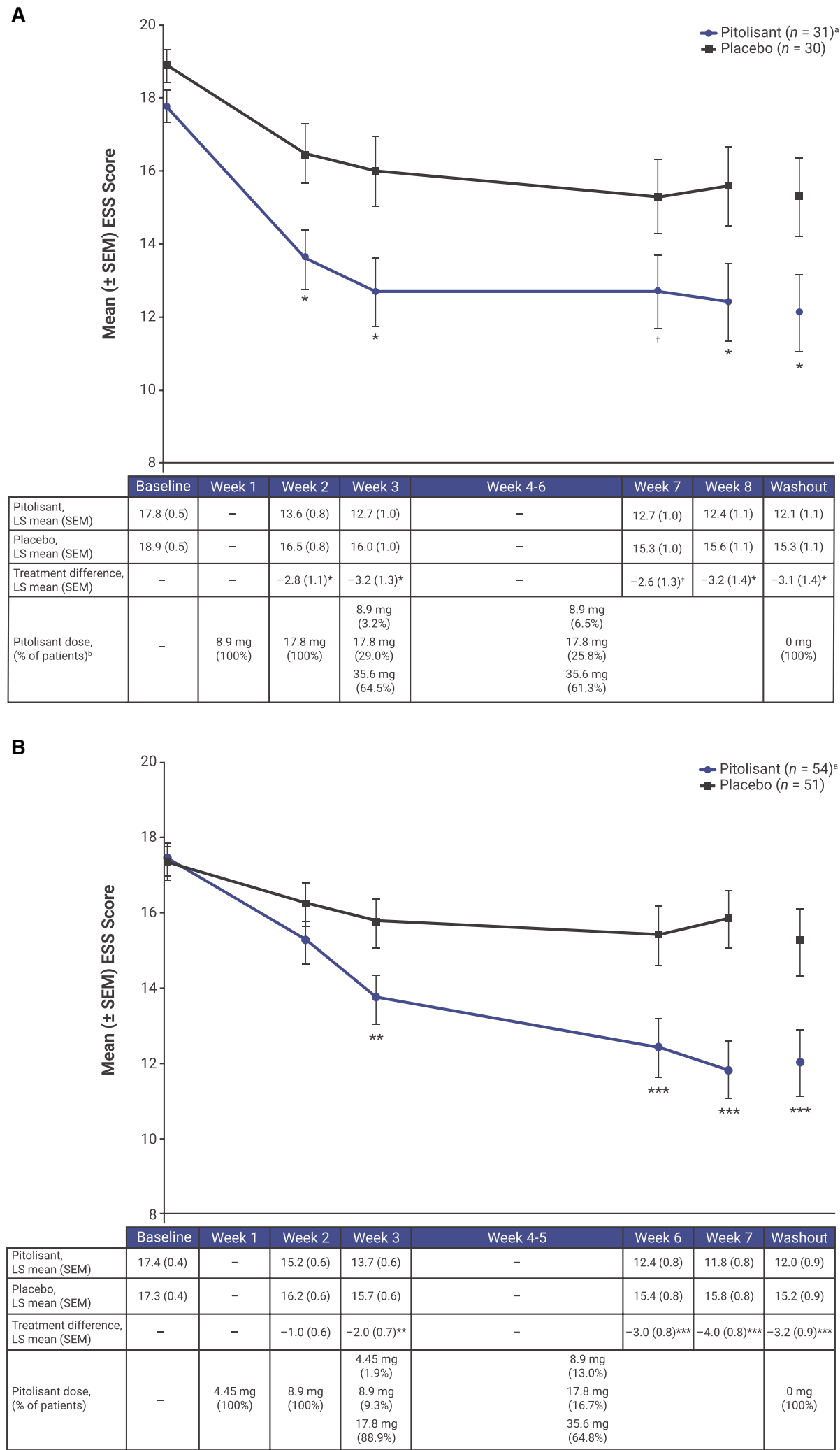

Fig. 1 Epworth Sleepiness Scale (ESS) scores over the course of treatment in A HARMONY 1 and B HARMONY CTP. Data are shown as mean [standard error of the mean (SEM)] at baseline and least-squares (LS) mean (SEM) at other timepoints with the last observation carried

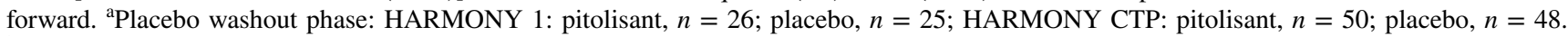
${ }^{b}$ Dosing information is based on the number of patients in the study at each timepoint; two patients did not receive a stable dose in HARMONY 1. $* p<0.05$ vs placebo; ${ }^{\dagger} p=0.05$ vs placebo; $* * p<0.01$ vs placebo; $* * * p<0.001$ vs placebo 
Fig. 2 Epworth Sleepiness Scale (ESS) responders over the course of treatment for response defined as an A ESS score reduction of $\geq 3$ and $\mathbf{B}$ final ESS score $\leq 10 . * p<0.05$ vs placebo; ${ }^{* *} p<0.01$ vs placebo; $* * * p<0.001$ vs placebo
A

HARMONY 1

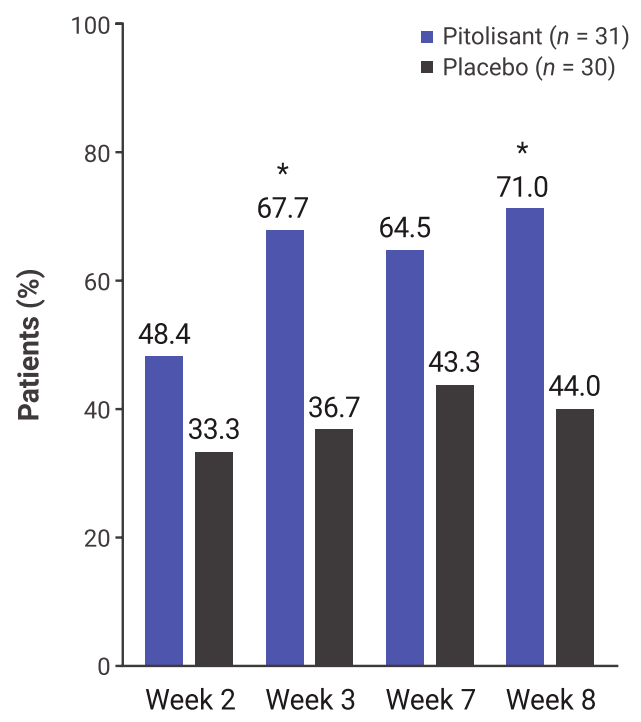

B

HARMONY 1

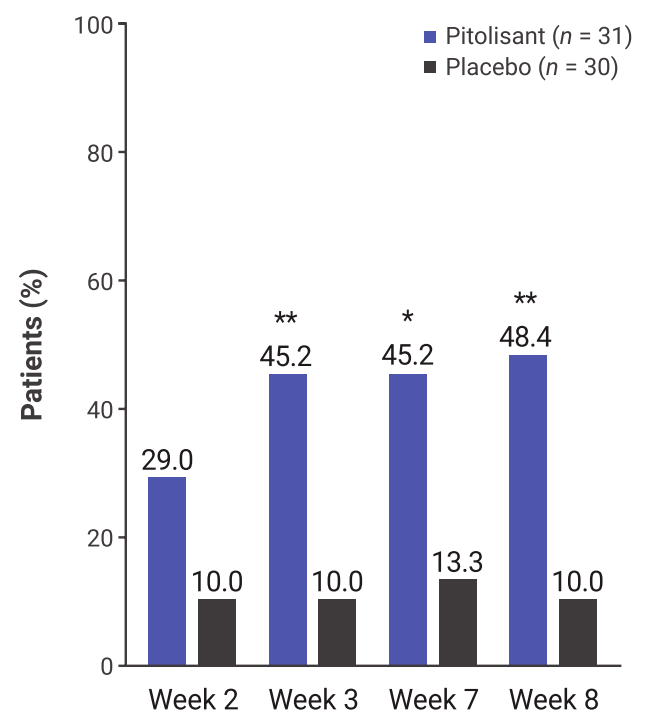

HARMONY CTP

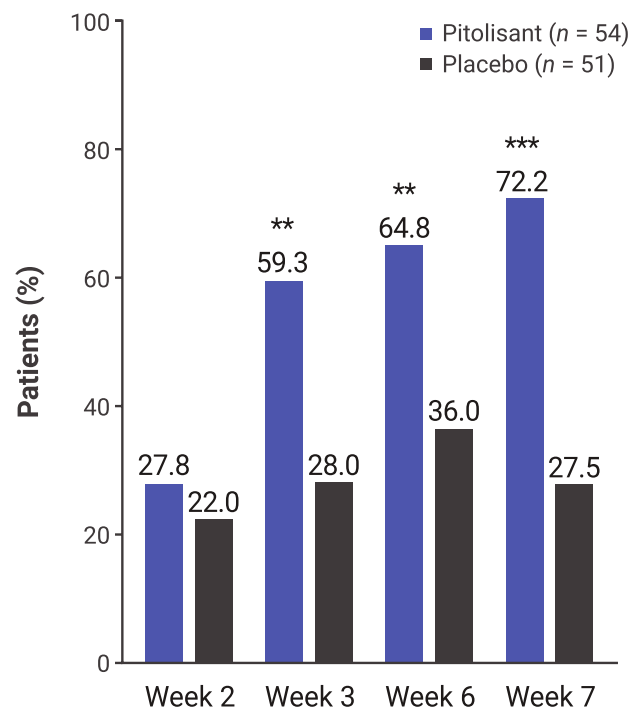

HARMONY CTP

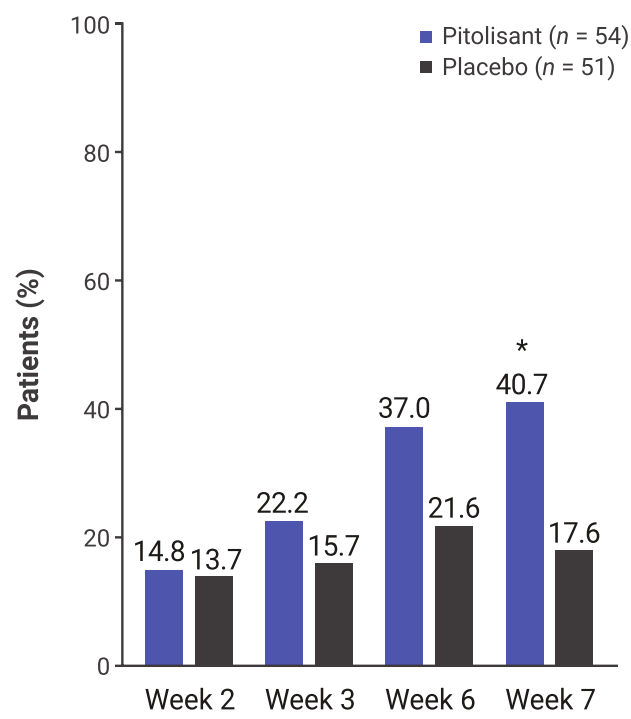

in HARMONY CTP. At the end of treatment, the LS mean change from baseline WRC was -6.5 for pitolisant vs -0.1 for placebo $(p<0.001)$. The percentage of cataplexy responders in HARMONY CTP was significantly greater with pitolisant vs placebo beginning at week 1 for a response defined as $\mathrm{a} \geq 25 \%$ reduction in WRC and at week 2 for a response defined as a $\geq 50 \%$ reduction in WRC (Fig. 5). On the CGI-C for cataplexy, more patients in HARMONY CTP were considered by the investigator to be "much" or "very much" improved with pitolisant vs placebo, with statistically significant differences between groups observed at weeks 6 and 7 (Fig. 6). In the subset of HARMONY 1 patients with cataplexy at baseline, the LS mean (standard error) change in WRC at the end of treatment (week 8) was - 6.7 (2.4) in the pitolisant group and +2.5 (2.8) in the placebo group; the LS mean (standard error) treatment difference was -9.2 (3.7) attacks per week $(p=0.035)$. In this relatively small subset of HARMONY 1 patients, a reduction in WRC was significantly greater with pitolisant relative to placebo beginning at week 5 [LS mean (standard error) treatment difference, -8.5 (3.5); $p=0.047]$. 
Fig. 3 Clinical Global Impression of Change for excessive daytime sleepiness over the course of treatment in

A HARMONY 1 and B HARMONY CTP. Response on the Clinical Global Impression of Change was defined by ratings of "much" or "very much" improved. a No Clinical Global Impression of Change data for one patient in the pitolisant group in HARMONY $1 . * p<0.05$ vs placebo
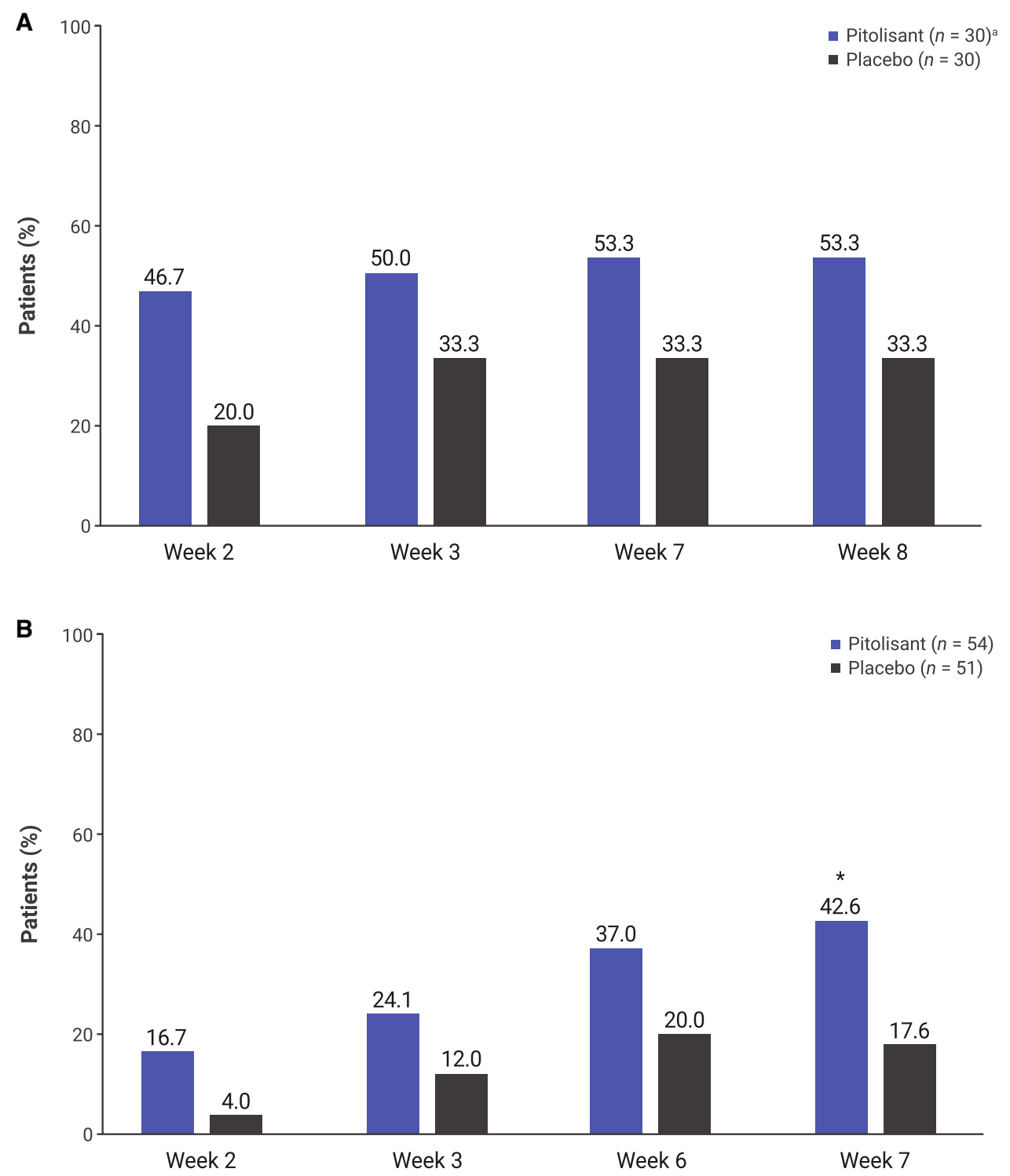

After 1 week of double-blind placebo washout, no significant difference in mean WRC was observed for pitolisant vs placebo in HARMONY CTP; the between-group difference in HARMONY 1 significantly favored pitolisant $(p=0.041)$. In the pitolisant group in HARMONY CTP, LS mean WRC was 11.7 at baseline, 4.2 at the last on-treatment assessment, and 7.1 after the placebo washout period. In the pitolisant group in HARMONY 1, LS mean WRC was 10.5 at baseline, 2.0 at the last on-treatment assessment, and 1.3 after the placebo washout period. Thus, there was no evidence of rebound cataplexy, and no patients in either study experienced status cataplecticus after pitolisant was discontinued.

\subsection{Adverse Events}

The most common AEs in pitolisant-treated patients (incidence $\geq 5 \%$ ) were headache ( $35.5 \%$ vs $20.0 \%$ with placebo), nausea
(6.5\% vs $3.3 \%)$, insomnia (6.5\% vs $0 \%)$, weight increase $(6.5 \%$ vs $6.7 \%)$, and nasopharyngitis (6.5\% vs $3.3 \%)$ in HARMONY 1 and headache $(9.3 \%$ vs $9.8 \%)$, nausea (5.6\% vs $0 \%)$, anxiety ( $5.6 \%$ vs $0 \%$ ), and irritability (5.6\% vs $2.0 \%)$ in HARMONY CTP. The frequency of AEs over the course of treatment is shown in Fig. 7. In HARMONY 1, AEs occurred primarily early in treatment and declined over time. For headache, the most common $\mathrm{AE}$ in pitolisant-treated patients, there were seven events in the pitolisant group during the first week of treatment, and no more than two events during any subsequent week. In HARMONY CTP, the number of AEs varied by week and was relatively low throughout the study. The majority of AEs were mild or moderate in intensity; severe AEs were reported for $6.5 \%$ of patients in the pitolisant group and $3.3 \%$ of patients in the placebo group in HARMONY 1 and for $1.9 \%$ and $0.0 \%$ of patients, respectively, in HARMONY CTP. Across the two studies, one patient receiving pitolisant (in HARMONY 


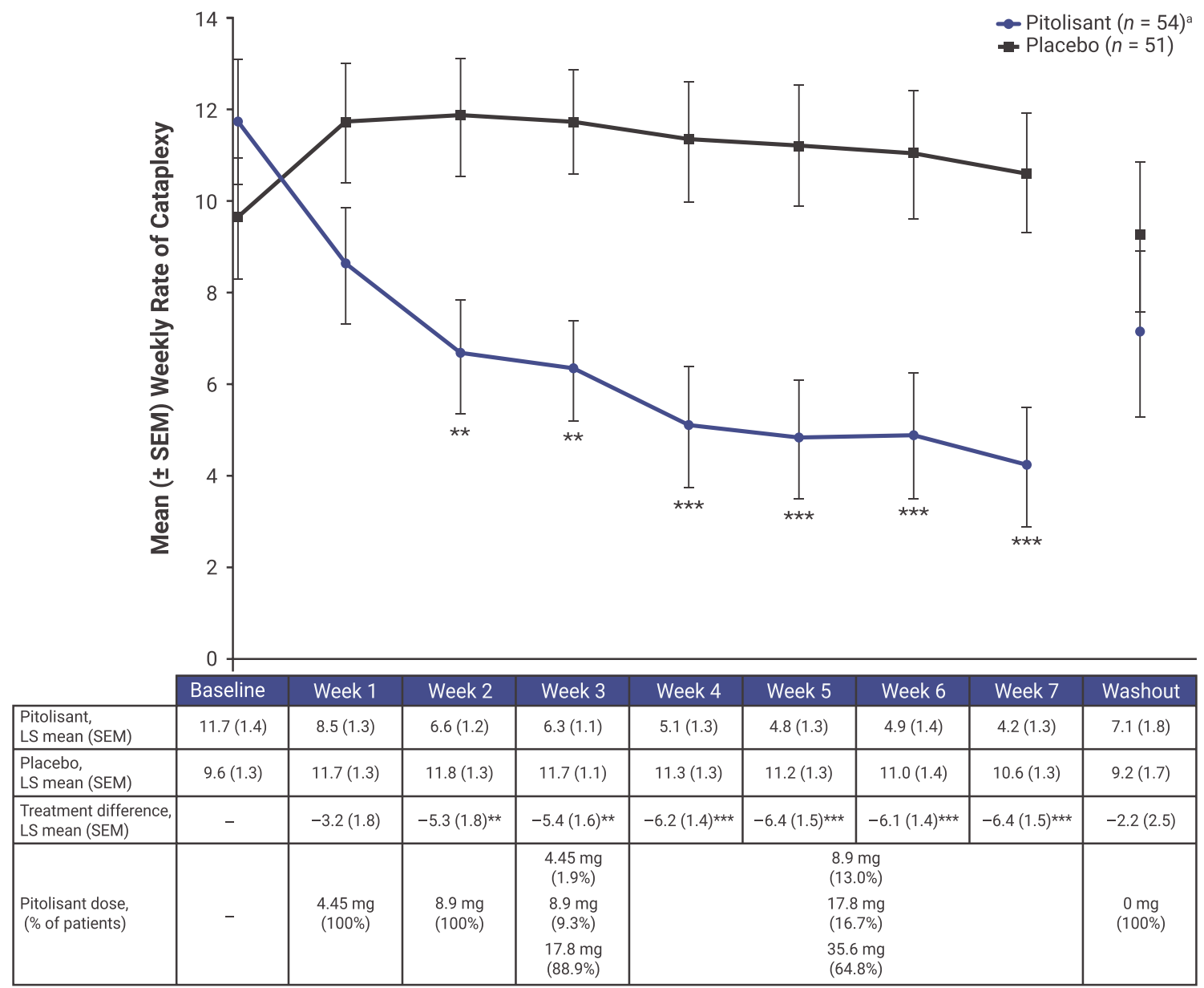

Fig. 4 Weekly rate of cataplexy attacks over the course of treatment in HARMONY CTP. Data are shown as mean (standard error of the mean $[\mathrm{SEM}]$ ) at baseline and least-squares (LS) mean (SEM) at other timepoints with the last observation carried forward. ${ }^{\text {a }}$ Placebo washout phase, pitolisant, $n=36$; placebo, $n=41$. ${ }^{*} p<0.01$ vs placebo; $* * * p<0.001$ vs placebo
CTP) and three patients receiving placebo (in HARMONY 1) discontinued study participation because of an AE.

\section{Discussion}

This analysis included data from two 7- to 8-week, placebocontrolled studies that permitted titration of pitolisant to the maximum recommended dose of $35.6 \mathrm{mg} /$ day in adults with narcolepsy $[32,33]$. In these studies, pitolisant was initiated at doses $(4.45 \mathrm{mg} /$ day or $8.9 \mathrm{mg} /$ day) that were expected not to have substantial benefit in the large majority of patients and then flexibly titrated to an individualized dose $(8.9 \mathrm{mg} /$ day, $17.8 \mathrm{mg} /$ day, or $35.6 \mathrm{mg} /$ day), which remained stable for the last 4 or 5 weeks of treatment. Findings from this analysis suggest that a response may be observed for some patients within the first or second week of initiating treatment, while for other patients several weeks may be required. In the overall patient population, onset of clinical response, as assessed using relatively sensitive measures (i.e., the ESS and WRC), became apparent during dose titration. Mean reductions in EDS and cataplexy were significantly greater with pitolisant relative to placebo after 2-3 weeks of treatment; initial statistical separation from placebo was observed for EDS when most patients were receiving a pitolisant dose of $17.8 \mathrm{mg} /$ day and for cataplexy when all patients were receiving $8.9 \mathrm{mg} /$ day. Mean ESS scores and cataplexy attack frequency continued to decrease in pitolisant-treated patients throughout these 7- to 8-week studies; from week 4 onward, the pitolisant dose was 35.6 $\mathrm{mg}$ /day in the majority of patients $(61 \%$ or $65 \%)$. There was no evidence of rebound EDS or cataplexy when pitolisant was discontinued.

Compared with these outcome measures, there was a longer time to the onset of response based on clinicians' perceptions. Whereas the ESS and WRC are continuous measures based on a sum of individual item scores (for the ESS [37]) or a weekly average of cataplexy attacks 
Fig. 5 Cataplexy responders over the course of treatment in HARMONY CTP for response defined as a $\geq 25 \%$ reduction in the $\mathbf{A}$ weekly rate of cataplexy attacks and $\mathbf{B}$ a $\geq 50 \%$ reduction in the weekly rate of cataplexy attacks. $* p<0.05$ vs placebo; $* * p<0.01$ vs placebo; $* * * p<$ 0.001 vs placebo
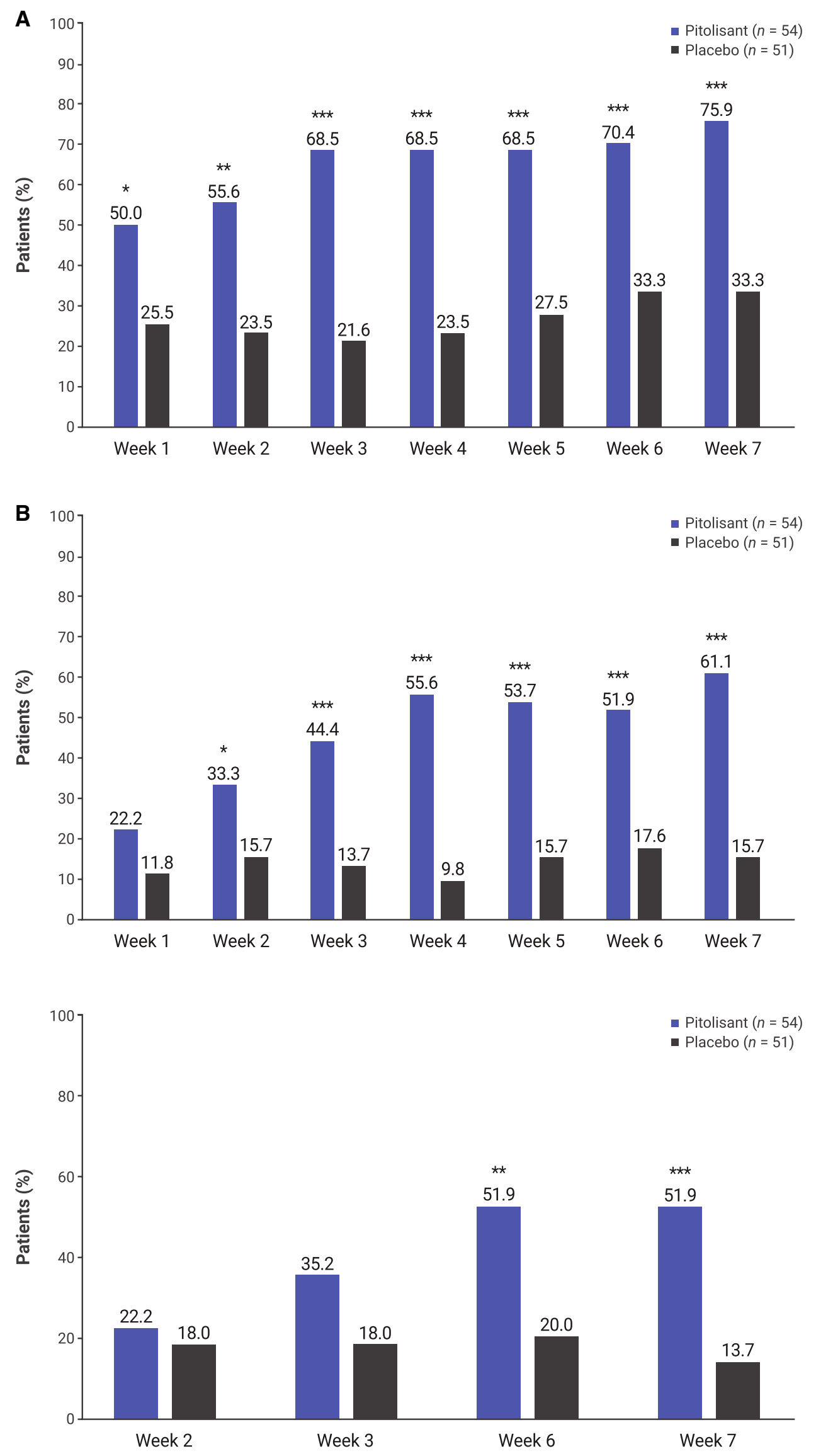

Fig. 6 Clinical Global Impression of Change for cataplexy over the course of treatment in HARMONY CTP. Response on the Clinical Global Impression of Change was defined by ratings of "much" or "very much" improved. ** $p<0.01$ vs placebo; $* * * p<0.001 \mathrm{vs}$ placebo 
Fig. 7 Frequency of adverse events over the course of treatment in A HARMONY 1 and B HARMONY CTP
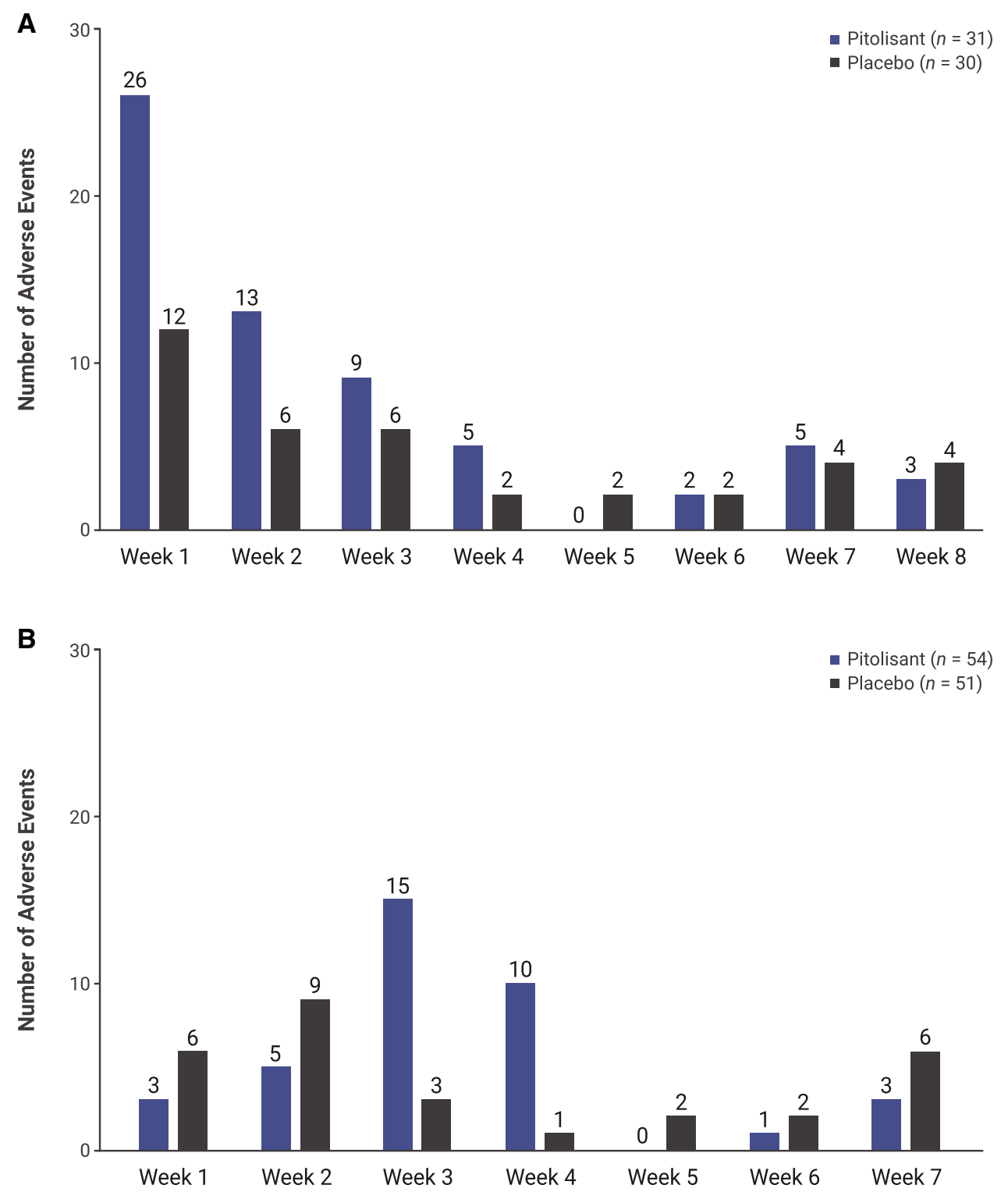

recorded in patient diaries (for the WRC), clinician reports were obtained from the CGI-C [38], a 7-point categorical scale with ratings that range from "very much worse" to "very much improved." Investigator-reported clinical response, defined as a rating of "much improved" or "very much improved" on the CGI-C, was significantly different for pitolisant vs placebo later in treatment (typically week 6 or 7), while treatment response was detected earlier when assessed via the ESS and patient reports of cataplexy attacks.

In this analysis, a clinically meaningful response was defined for EDS in two ways ( $a \geq 3$-point reduction from the baseline ESS score or a final ESS score $\leq 10$ ) and for cataplexy as a $\geq 25 \%$ or $\geq 50 \%$ reduction from baseline in WRC. The percentage of treatment responders was significantly greater for pitolisant vs placebo, with some variation in time to onset by study and by definition of response. The pattern of results also suggests variation in the timing of response related to the dose of pitolisant, as the response rates in the pitolisant group increased gradually over the course of treatment. After treatment with pitolisant for a total of 7 (HARMONY CTP) or 8 (HARMONY 1) weeks, a clinically meaningful response in EDS was observed in $71 \%$ or $72 \%$ of patients for a response defined as $\mathrm{a} \geq 3$ point reduction in the ESS score; $41 \%$ or $48 \%$ of patients had a final ESS score $\leq 10$. A clinically meaningful response in cataplexy, defined as a reduction in WRC of at least $50 \%$, was noted in $61 \%$ of pitolisant-treated patients at the last on-treatment assessment in HARMONY CTP; 76\% of patients had a WRC reduction of at least $25 \%$. The definitions of clinically meaningful response used in this analysis are consistent with clinical 
significance thresholds identified in the systematic literature review that supported the 2021 American Academy of Sleep Medicine clinical practice guideline on the treatment of central disorders of hypersomnolence [39].

The pattern of pitolisant-related AEs in HARMONY 1 is typical of many medications across therapeutic areas in that the frequency was highest during the first few weeks of treatment and declined over time. It is notable that only one pitolisant-treated patient discontinued study participation because of an $\mathrm{AE}[32,33]$; this finding indicates that discontinuation did not account for the reduction in $\mathrm{AE}$ frequency over time, suggesting that side effects may resolve with continued treatment. The relatively low frequency of AEs in HARMONY CTP does not have an obvious explanation but may be related to several factors including the titration schedule (pitolisant was initiated at the lowest possible dose [ $4.45 \mathrm{mg} / \mathrm{day}]$ ) and cultural/ ethnic differences in AE reporting between patients (who resided primarily in Eastern Europe in HARMONY CTP and Western Europe in HARMONY 1). Adverse events consistent with amphetamine-like withdrawal symptoms were assessed after study medication was discontinued, and the absence of withdrawal syndrome in the pitolisant group was noted in the original study reports [32, 33]. These findings are consistent with the results of a human abuse potential study, which found minimal to no abuse potential for pitolisant [31].

This analysis extends the findings of the original study reports by providing further information on the time course of clinical response to pitolisant. It is important to manage patients' expectations regarding improvements in their narcolepsy symptoms during treatment. In some patients, an initial clinical response may be experienced during dose titration with pitolisant, whereas in others a longer time receiving treatment may be required. The data suggest that the clinical response to pitolisant improves with an increased mean dose. Thus, in the absence of tolerability concerns, the overall risk-benefit profile of pitolisant is supportive of increasing the pitolisant dose (up to the recommended maximum dose of $35.6 \mathrm{mg} /$ day) to achieve maximum therapeutic effect. Another important finding is that, while standard measures of clinical response (ESS, number of cataplexy attacks) demonstrated an earlier onset of response to pitolisant (in the first 2-3 weeks), clinician assessment did not appreciate an overall benefit until week 7 or 8 . This would suggest that using the ESS or another measure of patient functioning earlier during treatment could offer a more sensitive approach to identifying the onset of response with pitolisant.

Limitations of this analysis include its post hoc nature and the relatively short duration of the placebo-controlled studies. However, study duration was adequate for evaluating the time to the onset and progression of clinical response with pitolisant. Results for cataplexy may have been confounded by the use of other anti-cataplectic medications, which were permitted at stable doses during the studies. In addition, it was not feasible to pool efficacy data across studies because of variations in study design, most notably differences in the titration schedule, duration of treatment, and assessment schedule. Data from the Maintenance of Wakefulness Test were not included in this analysis of time to the onset of response because the Maintenance of Wakefulness Test was administered at only two timepoints (baseline and end of treatment [week 7 or 8]) to minimize the burden on patients with regard to objective sleep testing throughout the trials. Because pitolisant was flexibly dosed based on efficacy and tolerability, a fixed-dose, parallel-group study could help to identify differences in dose-related response. Assessments of EDS and cataplexy over time were based on patient-report measures (the ESS and patient diaries) rather than objective measurement. Another patient-report measure (the Narcolepsy Severity Scale) assesses all five key narcolepsy symptoms (i.e., EDS, cataplexy, hypnagogic hallucinations, sleep paralysis, disrupted night-time sleep) [40] and may be useful in future studies.

\section{Conclusions}

Onset of a patient-reported clinical response, for reductions in both EDS and cataplexy, was generally observed within $2-3$ weeks of initiating treatment with pitolisant, which is during the recommended dose-titration period, although several additional weeks were required for the onset of a clinician-rated treatment response. Inter-individual variation was observed in the time to the onset of response, and findings support the clinical practice of increasing the pitolisant dose to $35.6 \mathrm{mg} / \mathrm{day}$, within the limits of tolerability, in order to achieve maximum therapeutic benefit. Overall, these data support the importance of evaluating the onset of clinical response early in treatment, as well as allowing adequate time for a treatment response to become apparent when managing patients with a chronic disorder such as narcolepsy.

Acknowledgements Technical editorial and medical writing assistance was provided under the direction of the authors by Nancy Holland, Ph.D., Synchrony Medical Communications, LLC, West Chester, PA, USA, and funded by Harmony Biosciences, LLC, Plymouth Meeting, PA, USA. WAKIX is a registered trademark of Bioprojet Europe, Ltd.

\section{Declarations}

Funding The studies from which the data analyzed in this paper were derived (NCT 1067222 and NCT 01800045) were funded by Bioprojet Pharma, Paris, France; the analysis reported in this paper and open access were funded by Harmony Biosciences LLC, Plymouth Meeting, PA, USA. 
Conflict of interest NFW reports serving as a consultant for Harmony Biosciences, LLC, Jazz Pharmaceuticals, and SleepScore Labs. CWD, DZ, and JMD are employees of Harmony Biosciences, LLC. BV is an employee of Rho. YD reports receiving funds for speaking, board engagements, and travel to conferences from Avadel/Flamel, Bioprojet Pharma, Harmony Biosciences, LLC, Idorsia, Jazz Pharmaceuticals, Takeda, Theranexus, and UCB Pharma. JCS is a co-founder of Bioprojet Pharma.

Ethics approval Both studies (NCT 1067222 and NCT 01800045) were conducted in accordance with the Good Clinical Practice guidelines of the International Council for Harmonisation and the ethical principles of the Declaration of Helsinki. Each study protocol was approved by an institutional review board or independent ethics committee at each study site.

Consent to participate All patients provided written informed consent before study enrollment.

\section{Consent for publication Not applicable.}

Data availability The datasets generated and/or analyzed for the current study are not publicly available.

\section{Code availability Not applicable.}

Authors' contributions NFW: data analysis and interpretation, and review and editing of the article; CWD: conceptualization, data analysis and interpretation, and review and editing of the article; DZ: conceptualization, data analysis and interpretation, and review and editing of the article; BV: data analysis, and review and editing of the article; JMD: conceptualization, data analysis and interpretation, and review and editing of the article; YD: data analysis and interpretation, and review and editing of the article; and JCS: conceptualization, data analysis and interpretation, and review and editing of the article. All authors provided input into the drafting of the manuscript, have reviewed and approved the final version, and agree to be accountable for the work presented in the manuscript.

Open Access This article is licensed under a Creative Commons Attribution-NonCommercial 4.0 International License, which permits any non-commercial use, sharing, adaptation, distribution and reproduction in any medium or format, as long as you give appropriate credit to the original author(s) and the source, provide a link to the Creative Commons licence, and indicate if changes were made. The images or other third party material in this article are included in the article's Creative Commons licence, unless indicated otherwise in a credit line to the material. If material is not included in the article's Creative Commons licence and your intended use is not permitted by statutory regulation or exceeds the permitted use, you will need to obtain permission directly from the copyright holder. To view a copy of this licence, visit http://creativecommons.org/licenses/by-nc/4.0/.

\section{References}

1. Bassetti CLA, Adamantidis A, Burdakov D, et al. Narcolepsy: clinical spectrum, aetiopathophysiology, diagnosis and treatment. Nat Rev Neurol. 2019;15:519-39.

2. Scammell TE. Narcolepsy. N Engl J Med. 2015;373:2654-62.

3. American Academy of Sleep Medicine. The international classification of sleep disorders. Darien: American Academy of Sleep Medicine; 2014.
4. Dauvilliers Y, Arnulf I, Mignot E. Narcolepsy with cataplexy. Lancet. 2007;369:499-511.

5. Maski K, Steinhart E, Williams D, et al. Listening to the patient voice in narcolepsy: diagnostic delay, disease burden, and treatment efficacy. J Clin Sleep Med. 2017;13:419-25.

6. Flores NM, Villa KF, Black J, et al. The humanistic and economic burden of narcolepsy. J Clin Sleep Med. 2016;12:401-7.

7. Ton TGN, Watson NF, Koepsell TD, et al. Narcolepsy and the sickness impact profile: a general health status measure. Sleep Sci. 2014;7:5-12.

8. Jennum P, Ibsen R, Petersen ER, et al. Health, social, and economic consequences of narcolepsy: a controlled national study evaluating the societal effect on patients and their partners. Sleep Med. 2012;13:1086-93.

9. Jennum P, Ibsen R, Kjellberg J. Long-term health and socioeconomic consequences of childhood and adolescent-onset of narcolepsy. Sleep Med. 2020;67:23-7.

10. Ohayon MM. Narcolepsy is complicated by high medical and psychiatric comorbidities: a comparison with the general population. Sleep Med. 2013;14:488-92.

11. Mahoney CE, Cogswell A, Koralnik IJ, et al. The neurobiological basis of narcolepsy. Nat Rev Neurosci. 2019;20:83-93.

12. Kornum BR, Knudsen S, Ollila HM, et al. Narcolepsy. Nat Rev Dis Primers. 2017;3:16100.

13. Latorre D, Kallweit U, Armentani E, et al. T cells in patients with narcolepsy target self-antigens of hypocretin neurons. Nature. 2018;562:63-8.

14. Barateau L, Dauvilliers Y. Recent advances in treatment for narcolepsy. Ther Adv Neurol Disord. 2019;12:1756286419875622.

15. Kallweit U, Bassetti CL. Pharmacological management of narcolepsy with and without cataplexy. Expert Opin Pharmacother. 2017;18:809-17.

16. Scammell TE, Jackson AC, Franks NP, et al. Histamine: neural circuits and new medications. Sleep. 2019;42:1-8.

17. Panula $P$, Nuutinen $S$. The histaminergic network in the brain: basic organization and role in disease. Nat Rev Neurosci. 2013;14:472-87.

18. Scammell TE, Arrigoni E, Lipton JO. Neural circuitry of wakefulness and sleep. Neuron. 2017;93:747-65.

19. España RA, Scammell TE. Sleep neurobiology from a clinical perspective. Sleep. 2011;34:845-58.

20. Thakkar MM. Histamine in the regulation of wakefulness. Sleep Med Rev. 2011;15:65-74.

21. Lin JS, Sergeeva OA, Haas HL. Histamine $\mathrm{H}_{3}$ receptors and sleepwake regulation. J Pharmacol Exp Ther. 2011;336:17-23.

22. Haas HL, Sergeeva OA, Selbach O. Histamine in the nervous system. Physiol Rev. 2008;88:1183-241.

23. Parmentier R, Ohtsu H, Djebbara-Hannas Z, et al. Anatomical, physiological, and pharmacological characteristics of histidine decarboxylase knock-out mice: evidence for the role of brain histamine in behavioral and sleep-wake control. J Neurosci. 2002;22:7695-711.

24. Schwartz JC. The histamine $\mathrm{H}_{3}$ receptor: from discovery to clinical trials with pitolisant. Br J Pharmacol. 2011;163:713-21.

25. Benarroch EE. Histamine in the CNS: multiple functions and potential neurologic implications. Neurology. 2010;75:1472-9.

26. Ligneau X, Perrin D, Landais L, et al. BF2649 [1-\{3-[3-(4-chlorophenyl) propoxy] propyl\} piperidine, hydrochloride], a nonimidazole inverse agonist/antagonist at the human histamine H3 receptor: preclinical pharmacology. J Pharmacol Exp Ther. 2007;320:365-75.

27. Lin JS, Dauvilliers Y, Arnulf I, et al. An inverse agonist of the histamine $\mathrm{H}_{3}$ receptor improves wakefulness in narcolepsy: studies in orexin-/- mice and patients. Neurobiol Dis. 2008;30:74-83. 
28. Dauvilliers Y, Siegel JM, Lopez R, et al. Cataplexy: clinical aspects, pathophysiology and management strategy. Nat Rev Neurol. 2014;10:386-95.

29. Burgess CR, Scammell TE. Narcolepsy: neural mechanisms of sleepiness and cataplexy. J Neurosci. 2012;32:12305-11.

30. Uguen M, Perrin D, Belliard S, et al. Preclinical evaluation of the abuse potential of pitolisant, a histamine $\mathrm{H}_{3}$ receptor inverse agonist/antagonist compared with modafinil. Br J Pharmacol. 2013;169:632-44.

31. Setnik B, McDonnell M, Mills C, et al. Evaluation of the abuse potential of pitolisant, a selective $\mathrm{H}_{3}$-receptor antagonist/inverse agonist, for the treatment of adult patients with narcolepsy with or without cataplexy. Sleep. 2020;43:1-12.

32. Dauvilliers Y, Bassetti C, Lammers GJ, et al. Pitolisant versus placebo or modafinil in patients with narcolepsy: a double-blind, randomised trial. Lancet Neurol. 2013;12:1068-75.

33. Szakacs Z, Dauvilliers Y, Mikhaylov V, et al. Safety and efficacy of pitolisant on cataplexy in patients with narcolepsy: a randomised, double-blind, placebo-controlled trial. Lancet Neurol. 2017;16:200-7.
34. Dauvilliers Y, Arnulf I, Szakacs Z, et al. Long-term use of pitolisant to treat patients with narcolepsy: Harmony III study. Sleep. 2019;42:1-11.

35. Wakix ${ }^{\circledR}$ (pitolisant) tablets, for oral use. Package insert. Plymouth Meeting, PA: Harmony Biosciences, LLC; 2020.

36. Wakix summary of product characteristics [SPC]. Available from: https://www.ema.europa.eu/documents/product-information/ wakix-epar-product-information_en.pdf. Accessed 4 Dec 2020.

37. Johns MW. A new method for measuring daytime sleepiness: the Epworth Sleepiness Scale. Sleep. 1991;14:540-5.

38. Guy W. ECDEU assessment manual for psychopharmacology revised. Rockville, MD: National Institute of Mental Health; 1976.

39. Maski K, Trotti LM, Kotagal S, et al. Treatment of central disorders of hypersomnolence: an American Academy of Sleep Medicine systematic review, meta-analysis, and grade assessment. J Clin Sleep Med. 2021;17:1895-945.

40. Dauvilliers Y, Beziat S, Pesenti C, et al. Measurement of narcolepsy symptoms: the Narcolepsy Severity Scale. Neurology. 2017;88:1358-65. 